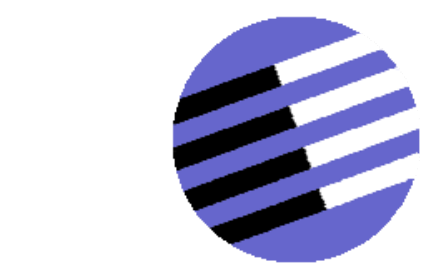

GOVERNANCE AND THE EFFICIENCY

OF ECONOMIC SYSTEMS

GESY

Discussion Paper No. 451

Hidden Benefits of Reward:

A Field Experiment on

Motivation and

Monetary Incentives

Ola Kvaløy*

Petra Nieken**

Anja Schöttner ***

* University of Stavanger, UiS Business School

** University of Bonn

*** University of Konstanz

December 2013

Financial support from the Deutsche Forschungsgemeinschaft through SFB/TR 15 is gratefully acknowledged. 


\title{
Hidden Benefits of Reward: A Field Experiment on Motivation and Monetary Incentives
}

\author{
Ola Kvaløy \\ Petra Nieken \\ Anja Schöttner*
}

December 2013

\begin{abstract}
We conducted a field experiment in a controlled work environment to investigate the effect of motivational talk and its interaction with monetary incentives. We find that motivational talk significantly improves performance only when accompanied by performance pay. Moreover, performance pay slightly reduces performance unless it is accompanied by motivational talk. These effects also carry over to the quality of work. Performance pay alone leads to more mistakes. Adding motivational talk makes the difference. In treatments with performance pay, motivational talk increases output by about 20 percent and reduces the ratio of mistakes by more than 40 percent.
\end{abstract}

JEL Classification: C93, M52, J33

Keywords: Verbal Motivation, Performance Pay, Field Experiment

\footnotetext{
${ }^{*}$ Kvaløy: University of Stavanger, UiS Business School, 4036 Stavanger, Norway (e-mail: ola.kvaloy@uis.no); Nieken: University of Bonn, Institute for Applied Microeconomics, Adenauerallee 24-42, 53113 Bonn, Germany (e-mail: petra.nieken@unibonn.de); Schöttner: University of Konstanz, Department of Economics, PO Box 144, 78457 Konstanz, Germany (e-mail: anja.schoettner@uni-konstanz.de). We thank Stefano DellaVigna, Robert Dur, Uri Gneezy, Sebastian Goerg, Heike Henning-Schmidt, Sebastian Kube, Mari Rege, Gaute Torsvik, Bertil Tungodden, the participants of the ESA World Meeting in Zurich, the Nordic Conference on Behavioral and Experimental Economics in Stockholm, the GEABA Meeting in Magdeburg, the Stavanger Workshop in Behavioral Economics, the GfeW Meeting in Helmstedt, the CESifo Area Conference in Behavioral Economics in Munich, and seminar participants at Erasmus University Rotterdam, Norwegian School of Economics, University of Karlsruhe, University of Konstanz, University of Munich, University of Regensburg, and University of Trier for helpful comments and suggestions. Financial support from the German Research Foundation (DFG), in particular SFB TR 15, and from the Norwegian Research Council is gratefully acknowledged.
} 


\section{Introduction}

There is ample evidence that monetary incentives are not always beneficial. In fact, monetary rewards, like bonuses or piece rates, can sometimes induce worse performance. Psychologists refer to this as a "hidden cost of reward:" performance pay crowds out the workers' intrinsic motivation, in particular by undermining workers' confidence in their own abilities or in the value of the rewarded task (Deci, 1975; Lepper and Greene, 1978). ${ }^{1}$ But intrinsic motivation is not only affected by monetary rewards. Firms spend resources in order to facilitate and evoke the intrinsic motivation of their workforce, for example by paying and developing managers and leaders with motivational skills. ${ }^{2}$ So a central question is, will performance pay undermine a leader's effort in motivating her workers? Or could monetary incentives instead complement and enhance the effect of a leader's motivational efforts?

Theory does not provide a clear answer to this. One conjecture is that, when performance pay undermines intrinsic motivation, it will also undermine a leader's attempt to evoke the workers' intrinsic motivation. But recent theoretical work shows that crowding out can be due to some informational asymmetries. If agents are uncertain about their own ability, the value of the task, or the character of the principal, then material incentives alone might create a negative signal that lowers the agents' intrinsic motivation (Bénabou and Tirole 2003, 2006; Ellingsen and Johannesson, 2008). ${ }^{3}$ An implication is that, if the information asymmetry is resolved, performance pay improves performance rather than backfires. Related to this, Ellingsen and Johannesson (2008) remark that the negative signaling effect of material incentives might not extend to a situation where the principal has multiple signals available. Our conjecture is that motiva-

\footnotetext{
${ }^{1}$ A seminal contribution is Deci (1971), who shows that some tasks may have own inherent rewards, making external monetary rewards unnecessary or even detrimental to effort. Using controlled laboratory and field experiments, economists have demonstrated negative effects of monetary incentives in a variety of settings; see Frey and OberholzerGee (1997), Gneezy and Rustichini (2000a,b), Bohnet et al. (2001), Fehr and Rockenbach (2003), and Pokorny (2008). See also Gneezy and Rey-Biel (2011) for a nice review of this literature.

${ }^{2}$ See, e.g., Sims (1998) on management training and leadership development.

${ }^{3} \mathrm{~A}$ compensation scheme may also signal a social norm, which the agent might prefer to follow (Sliwka, 2007).
} 
tional efforts by a leader telling her employees how diligent they are and how important their work is, can serve as such an extra signal, which potentially changes the perception of performance pay. Similarly, performance pay might change the perception of motivational efforts. For example, a leader's visionary speeches or motivational pep talks might be perceived as more credible (and not just cheap talk) if he or she is willing to "put money behind the words."

In this paper, we present results from a field experiment designed to investigate the interaction between performance pay and motivational efforts. We focus on "motivational talk," words that potentially inspire workers to exert extra effort. On behalf of a research group at the University of Bonn, we hired students to enter data from ice hockey game reports into a database. The students, who were unaware of their participation in an experiment, were randomly assigned to one out of four treatments: with or without performance pay and with or without motivational talk (motivation, for short). In treatments with performance pay, subjects received a small piece rate (10 euro cents) on top of a fixed payment (20 euro) for each game report they were able to enter into the database. In treatments with motivation, subjects were exposed to simple motivational sentences in addition to a plain explanation of the task they were about to do.

We find that motivational talk significantly improves performance only when accompanied by performance pay. Moreover, performance pay reduces performance unless it is accompanied by motivational talk. This also carries over to the quality of work. Performance pay alone leads to more mistakes. Adding motivational talk, however, makes the difference. Although subjects were not rewarded for quality, performance pay has a positive effect on quality if it is accompanied by motivational talk. Hence, we find what we can call a hidden benefit of monetary rewards: complementarity between performance pay and motivational talk. The effects are strong. In treatments with performance pay, motivational talk increases output by about 20 percent and reduces the ratio of mistakes by more than 40 percent.

Related literature: While economists have investigated motivation primarily through the lens of monetary incentives, psychologists have been more interested in the effect of non-monetary motivators such as recogni- 
tion, attention, and verbal feedback. An early lab experiment is Deci and Ryan (1971) showing that provision of praise increases students' willingness to work on a puzzle. Cameron and Pierce (1994) provide a survey of the subsequent lab-experimental literature. Stajkovic and Luthans (2003) give an overview of experimental field studies on performance-dependent recognition and report strong, positive effects in a variety of workplace contexts. More recent papers include Grant and Gino (2010), who study experimentally how a manager's verbal expression of gratitude affects employees' effort, and Kosfeld and Neckermann (2011) and Bradler et al. (2013), who use field experiments to investigate how social recognition affects employee performance. All find substantial positive effects. ${ }^{4}$ Our paper differs from this literature in two respects: First, we focus on non-contingent verbal motivation prior to work instead of performance feedback and contingent non-monetary rewards. Second, we are particularly interested in the interaction between verbal motivation and monetary incentives, which to our knowledge has not been systematically investigated. ${ }^{5}$

While the formal literature on intrinsic motivation and crowding out (cited above) provides some possible implications for the effect of nonmonetary motivation, there are only a few papers that explicitly address the optimal interaction between non-monetary motivation and monetary rewards. Marino and Zábojnik (2008) study the trade-off between workrelated perks and incentive provision, and Dur et al. (2010) analyze how attention paid by the principal to the agent affects optimal incentive contracting. Furthermore, Kvaløy and Schöttner (2013) analyze optimal incentive provision when a motivator can exert motivational effort to reduce the effort costs of an agent. But none of these papers consider the direct, negative effects of performance pay. In order to explicitly address the potential signalling effect of both performance pay and motivational talk, we

\footnotetext{
${ }^{4}$ Related is also the experimental literature examining how feedback and communication can help resolve coordination problems. In particular, see Brandts and Cooper (2007) who study how managers can use both monetary incentives and communication in order to help employees coordinate on high effort-levels.

${ }^{5} \mathrm{In}$ addition to the experimental literature, there is also a large body of literature on organizational behavior that uses survey data in order to investigate employees' motivation and experience with performance pay. Some report a positive relationship between performance pay and intrinsic motivation. Babakus et al. (1996), Baldauf et al. (2002), Miao and Evans (2007), and DelVecchio and Wagner (2011) all find that more incentive pay leads to higher levels of intrinsic motivation among sales people.
} 
adopt a variant of Bowles and Polanía-Reyes (2012) model on monetary incentives and social preferences for our theoretical framework.

The rest of the paper is organized as follows: Section 2 contains a model on the interaction between motivational talk and monetary incentives. In Section 3, we present the experimental design and procedure, while in Section 4 we present the results. Section 5 concludes.

\section{Model}

In this section, we present a simple principal-agent model with moral hazard to analyze how performance pay and motivational talk of the principal might affect the agent's effort choice and consequently his performance. We assume that the risk-neutral agent produces a verifiable output $y$. Output is a function of the agent's non-observable effort $e \geq 0$ and the realization of a non-observable random variable $\varepsilon$. Specifically, we assume that $y=\varepsilon e$ with $\varepsilon \geq 0$ and $E[\varepsilon]=1$. The agent's private effort costs are $c(e)=\frac{e^{2}}{2}$. The principal pays the agent a fixed wage $s$ and a piece rate $r \geq 0$ conditioned on output $y$. In addition, the principal can motivate the agent by exerting motivational effort (in the form of motivational talk) $a \geq 0$. Motivational effort is costly to the principal, e.g., due to opportunity costs of time.

We assume that the principal is better informed about the production environment than the agent. As a result, the principal's choice of monetary incentives and motivational effort constitutes a signal that allows the agent to better assess the circumstances of production. For example, performance pay might affect the agent's assessment of the task difficulty or his task-specific ability (Bénabou and Tirole, 2003) or of the principal's character and whether she is worth impressing (Bénabou and Tirole, 2006; Ellingsen and Johannesson, 2008). Similarly, motivational talk can influence the agent's perception of the task, his ability, or the principal's type. For example, if a principal takes the time to engage in motivational talk, this might signal that the work is important or that she has social preferences or cares about the agent. In theory, however, motivational talk could also indicate that the task is likely to be unattractive, because the principal sees the need for some extra motivation.

To account for the potential signalling aspects of performance pay and 
motivation, we consider a variant of the state-dependent utility function proposed by Bowles and Polanía-Reyes (2012). Because these authors focus on monetary incentives, we extend their utility function to allow for a potential impact of motivational talk. We thus assume that the agent's expected utility is

$$
s+r E[y \mid e]-c(e)+e\left(\mu_{0}+\mu_{r} r+\mu_{a} a+\mu_{a r} a r\right),
$$

with the parameters $\mu_{0} \geq 0$ and $\mu_{r}, \mu_{a}, \mu_{a r} \in \mathbf{R}$. The parameter $\mu_{0}$ measures the agent's baseline intrinsic motivation, i.e., his marginal utility from exerting effort in the absence of motivation and performance pay. The remaining parameters reflect potential crowding effects of the piece rate $r$ and the motivational effort $a$, respectively, due to the signal content of these instruments. ${ }^{6}$ When the principal employs either performance pay or motivational talk only, the crowding effects are described by the parameters $\mu_{r}$ and $\mu_{a}$, respectively. When performance pay and motivation are used simultaneously, however, the signal content of either instrument might change, so that the crowding effects are non-separable in performance pay and motivation. This is the case if $\mu_{a r}$ is different from zero.

The timing is as follows: The principal first announces the compensation scheme $(s, r)$. Afterwards, she exerts motivational effort $a$. The agent observes $a$ and exerts effort $e$. Finally, $y$ is realized and the principal pays the agent. Now consider the stage where the agent chooses effort to maximize his expected utility (1). The optimal effort choice $e^{*}(a, r)$ thus is

$$
e^{*}=r+\mu_{0}+\mu_{r} r+\mu_{a} a+\mu_{a r} a r .
$$

From (2), we can derive the agent's incentive responsiveness $e_{r}^{*}$ and his "motivation responsiveness" $e_{a}^{*}$,

$$
e_{r}^{*}=1+\mu_{r}+\mu_{a r} a \text { and } e_{a}^{*}=\mu_{a}+\mu_{a r} r .
$$

By $(2)$, if $\mu_{0}>0$, the agent exerts effort even if the principal employs

\footnotetext{
${ }^{6}$ Variations in the fixed payment $s$ might also provide a signal on the production environment. However, we abstract from this possibility because the fixed payment is identical in all treatments of our experiment.
} 
neither performance pay nor motivational talk $(r=a=0)$. Now assume that the principal introduces a piece rate in addition to the fixed wage $(r>0$ and $a=0)$. By (3), the agent might respond by an effort increase $\left(e_{r}^{*}>0\right)$ or a weak decrease in effort $\left(e_{r}^{*} \leq 0\right)$. In the former case, the piece rate either constitutes a favorable signal on the production environment and thus crowds in the agent's effort $\left(\mu_{r}>0\right)$, or the piece rate is an unfavorable signal that crowds out effort, but this effect does not dominate the marginal utility from a larger expected monetary payoff $\left(0>\mu_{r}>-1\right)$. In contrast, the agent weakly reduces his effort under the piece rate if the crowding out effect is sufficiently strong $\left(\mu_{r} \leq-1\right)$. If the principal engages in motivational talk in addition to the fixed wage $(r=0$ and $a>0)$, the agent increases his effort if and only if $\mu_{a}>0$, i.e., motivational talk provides a favorable signal on the production environment.

We are particularly interested in the interaction between performance pay and motivational talk. From (3), we obtain that the two instruments can be substitutes $\left(e_{a r}^{*}=\mu_{a r}<0\right)$ as well as complements $\left(e_{a r}^{*}=\mu_{a r}>\right.$ $0)$. First, consider the case $e_{a r}^{*}<0$. It implies that, if motivational talk crowds in effort $\left(\mu_{a}>0\right)$, incentives will make motivational talk less fruitful. For example, motivational talk alone might be a favorable signal on the principal's characteristics, which is, however, counteracted by a piece rate. If, on the other hand, motivational talk provides a signal that crowds out effort $\left(\mu_{a}<0\right)$, this signal will be amplified by performance pay. For instance, the agent might assume that the principal exerts motivational effort to talk him into an unpleasant job. In both situations, the interaction of performance pay and motivational talk is detrimental to effort, which can be interpreted as a "hidden cost of reward."

In the more interesting case $\mu_{a r}>0$, a piece rate enhances the effectiveness (or reduces the defectiveness) of motivational talk, which can be termed "hidden benefit of reward." For example, without performance pay, motivational talk might not be credible and thus only be seen as cheap talk, while performance pay shows that the principal is willing to "put money behind the words." Moreover, $\mu_{a r}>0$ could also mean that motivational talk counteracts a potentially unfavorable signal of performance pay $\left(\mu_{r}<0\right)$. The principal might even be able to use motivational talk to resolve the problem of asymmetric information on the production environment, that 
is responsible for the crowding out effect of performance pay. The latter is then completely eliminated by motivational talk (i.e., $\mu_{r}+\mu_{a r} a=0$ ).

Our rather general theoretical framework can tell us something about potential interaction effects between motivational talk and performance pay, but it cannot provide us with any obvious hypothesis in this respect. We will thus investigate the sign of $e_{a r}$ empirically without stating any formal hypothesis.

\section{Experimental design and procedure}

\subsection{General description}

We conducted a field experiment to study the interaction between performance pay and motivational talk. On behalf of a research group at the University of Bonn, we hired students to enter data from official game reports of the German ice hockey league into a database (Microsoft Excel spreadsheet). This database needed to be extended for an ongoing research project. The task was rather simple, but it also required a certain degree of care and attention from the subjects. ${ }^{7}$

The subjects were not aware of their involvement in an experiment. We recruited them by using a written announcement, which we advertised on the university's job market website and the electronic newsletters of different faculties. Additionally, we distributed flyers in the university's two main cafeterias for two weeks in a row. The announcement advertised a onetime job opportunity for entering data for two hours. The compensation was stated as approximately 10 euro per hour, which is slightly higher than the hourly wage of a student assistant.

Altogether 203 students applied. The students were randomly assigned to the treatments, taking into account that a few of them stated time restrictions in their application. We invited them via e-mail and asked them to confirm their appointment. Moreover, we sent a reminder two days before a session, with the date and time of their appointment. The

\footnotetext{
${ }^{7}$ Similar data entry tasks are frequently used in field experiments because they allow measurement of performance and quality without frequently monitoring the subjects (see, e.g., Henning-Schmidt et al., 2010; Kosfeld and Neckermann, 2011; and Kube et al., 2012). Monitoring might interfere with incentives from performance pay.
} 
experiment was conducted on four days in November and December 2012 at the University of Bonn. We executed two sessions for each of our four treatments with 17 to 20 subjects each (some subjects failed to show up for work), yielding a total of 139 observations for our analysis (46 males and 93 females $)^{8}$

To establish a work environment that was as natural as possible, the sessions were not conducted in the BonnEconLab but in a computer pool at the University of Bonn. Upon arrival, each subject was randomly placed in front of a computer terminal. On the desk next to the computer was a box containing printed, official game reports from the German ice hockey league. Each subject at a certain terminal, e.g., terminal 1, had to enter the same data as the subjects of other sessions sitting at the same terminal, i.e., the data entered was identical for each terminal, but different between the terminals of one session. Hence, the difficulty of the data entering process was the same in all sessions. The subjects were able to see each other. However, because the printed reports were stored in a box and the finished ones had to be put in another box next to them, they could not observe how many reports the other subjects entered. The task was explained by the experimenter at the beginning of each session. The experimenter followed a detailed protocol and read the explanations aloud, instead of reciting them from memory, to ensure that the wording was exactly the same in each session. She also presented powerpoint slides with screenshots of the spreadsheet to demonstrate which data had to be entered in a certain cell. This procedure took about 15 minutes. To rule out a possible influence on the subjects' behavior, the experimenter did not change her physical appearance (clothes, hair, etc.) from one session to the next.

The subjects had the opportunity to ask clarifying questions before the working period started. In all sessions some subjects asked how to adjust the zoom, the width of a cell, and how they could scroll through the document. They also asked how they could store the spreadsheet to save the results from time to time. After answering all questions in plenary, the experimenter left the room. We chose to leave the subjects alone during the 90-minute working period to rule out possible effects of monitoring. The

\footnotetext{
${ }^{8} 143$ students participated in the experiment. Due to hardware failure, however, the data was not stored completely for 4 participants.
} 
subjects were informed that the experimenter would be available next door for questions. The subjects knew that the experimenter would interrupt them after 45 minutes and ask them to upload the data to a central storage to avoid data loss.

\subsection{Treatments}

We employed a $2 \times 2$ design: with or without performance pay and with or without motivational talk, as illustrated in Table 1.

Table 1: Overview treatments

\begin{tabular}{lcc}
\hline \hline & Fixed payment & Performance payment \\
\hline Neutral talk & fixed treatment & pfp treatment \\
Motivational talk & fixed-moti treatment & pfp-moti treatment \\
\hline
\end{tabular}

In the two treatments with motivational talk (called fixed-moti and pfpmoti) subjects were exposed to simple motivational sentences in addition to a plain explanation of the task they were about to do. Except for these sentences, the instructions for all treatments were identical. ${ }^{9}$ The motivational sentences were as follows:

Beginning of instructions: Welcome to the CIP Pool of the Juridicum. I am glad that you decided to assist us by recording the data for a research project. In the framework of a large research project, we analyze sports data, in this case ice hockey, to investigate the behavior of teams and competitive situations. We are certain that you, as competent students of the University of Bonn, are able to record the data quickly and precisely.

End of instructions: I know that the work might be exhausting and tiring, but I also know that you are diligent students who are able to concentrate on these kinds of tasks for longer periods. If you decide to leave the room, please try to be quiet to avoid disturbing your colleagues. You are welcome to adjust the screen to your convenience or to alter the zoom in the excel

\footnotetext{
${ }^{9}$ Complete instructions translated into English can be found in the appendix.
} 
sheet. If there are no more questions, let's start working! In addition, when the subjects were interrupted to upload the data after 45 minutes, they were told: Keep up the good work!

These are moderate motivational sentences, representing key aspects of motivational leadership (see, e.g., Robbins and Judge (2013), for an overview). The "leader"-represented by the experimenter-expresses purpose and meaning, positive expectations, and sensitivity to the workers' needs. ${ }^{10}$ Subjects might deduce from these words that they are participating in a valuable project and are likely to do a good job, which will be to the benefit of a (likable) employer. In contrast, the neutral talk says nothing about the purpose of the work or the employer's expectations.

In treatments with performance pay (called pfp and pfp-moti), subjects received a small piece rate (10 euro cents) on top of the fixed payment (20 euro) for each game report they entered into the spreadsheet. This compensation scheme resulted in performance pay of approximately 10 percent on top of fixed pay, which is quite common in practice. We informed the subjects at the end of the instructions about their payment.

While we did not control for the quality of the entered data, we did, however, check the number of entered game reports at the end of the working period to determine the payment in the performance-pay treatments. We conducted the fixed-payment sessions before the performance-pay sessions to avoid the expectations of being paid by performance. On average, subjects earned 21.06 euro (20 euro in the treatments with fixed pay and 22.17 euro in the treatments with pay for performance).

\section{Results}

Our key performance variable is the number of entered games in the spreadsheet (i.e., quantity). ${ }^{11}$ In a second step, we also analyze the effects on the

\footnotetext{
${ }^{10}$ With respect to the latter, we did not want motivational talk to make the task easier by improving the subjects' technological competence. Hence, information about screen and zoom adjustments were made available in each treatment. However, it was made up front in the motivational-talk treatments (showing sensitivity to workers' needs), while the information in the neutral-talk treatment was given either as a response to or after practical questions by subjects (in plenary) before they started working.

${ }^{11}$ To take into account that the number of filled cells varied between the games, we also used the number of filled cells as a robustness check. The results remain qualitatively
} 
Figure 1: Average performance over treatments

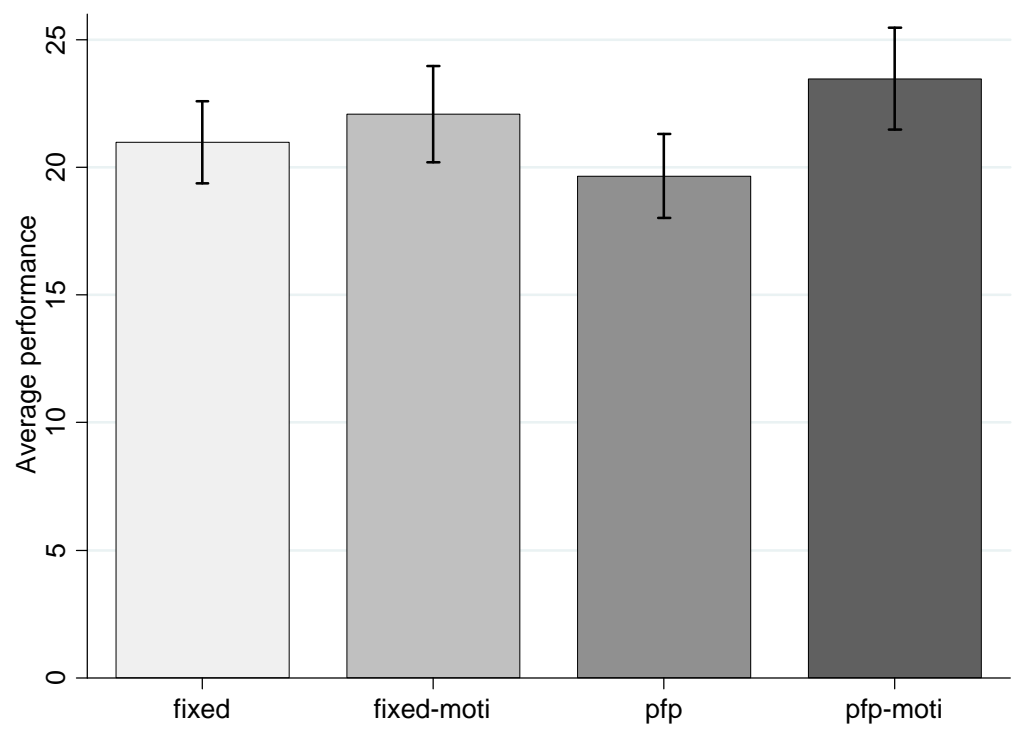

quality of the work.

The bar chart in Figure 1 shows the average performance in the four treatments, as well as the 95 percent confidence intervals. The subjects entered more games in the fixed-moti (mean 22.08) than in the fixed treatment (mean 20.97), but this difference is not statistically significant (MannWhitney $\mathrm{U}$ test, $p=0.377) .{ }^{12}$ However, adding motivation to pay for performance increases performance significantly (Mann-Whitney $\mathrm{U}$ test, $p=0.004)$. The mean performance is 19.66 in the pfp-treatment and 23.47 in the pfp-moti treatment.

This indicates that performance pay and motivational talk complement each other. But, in order to investigate whether performance pay enhances the effect of motivational talk (and vice versa), one has to study the interaction between the variables. We thus estimate a linear regression with an interaction term. Motivational talk $a$ and performance pay $r$ are the independent variables (main effects), and the number of entered games $y$

the same and can be obtained from the authors upon request.

${ }^{12}$ All Mann-Whitney U tests are two-sided. 
is the dependent variable:

$$
y_{i}=\beta_{0}+\beta_{1} a_{i}+\beta_{2} r_{i}+\beta_{3} a_{i} r_{i}+\text { controls }+\varepsilon_{i}
$$

Here, $a_{i}=1$ if subject $i$ was exposed to motivational talk (i.e., participated in fixed-moti or pfp-moti treatment), while $r_{i}=1$ if subject $i$ received performance pay (i.e., participated in pfp or pfp-moti treatment). Hence, $\beta_{1}$ shows the effect of motivation without performance pay, $\beta_{2}$ shows the effect of performance pay without motivation, while $\beta_{3}$ estimates the interaction between motivation and performance pay. We added additional control variables (gender, age, a dummy indicating if the subject is enrolled in economics, and session time ${ }^{13}$ ) and calculated robust standard errors clustered on sessions. ${ }^{14}$

The results of the regressions are reported in Table 2. ${ }^{15}$ Column (1) shows the results for overall performance. We first see that motivational talk alone has no significant effect on overall performance. Moreover, performance pay alone actually has a significantly negative effect on performance when it is not accompanied by motivational talk. However, the experiment identifies what can be termed a "hidden benefit of reward:" The interaction coefficient $y_{a r}=\beta_{3}$ is positive and significantly different from zero, i.e., motivational talk and performance pay are complements. This implies that performance pay makes motivational talk more efficient and that motivational talk improves the effect of performance pay. In the appendix we also present regressions on treatment effects, showing that subjects in the pfp-moti treatment perform significantly better than subjects in any of the other three treatments (Table A1).

Next, we analyze the performance during the first 45 minutes of the working period (first half) and the performance during the second 45 minutes

\footnotetext{
${ }^{13}$ Each treatment had one morning session and one afternoon session.

${ }^{14}$ We checked whether the observable characteristics of the participants such as gender, age, and course of study are balanced across treatments with Pearson Chi Square and Kruskal Wallis tests. Overall, the characteristics are well balanced, with the exception of participants who are enrolled in economics. They participated more often in the fixed-moti than in the other treatments.

${ }^{15}$ Note that the results remain qualitatively the same if we take into account that our dependent variable consists of non-negative integers and resembles count data and estimate negative binomial regressions instead of OLS. The results can be obtained from the authors upon request.
} 
(second half) separately. This enables us to see whether the behavior of the subjects changed over the course of the working period. Recall that the subjects were interrupted after 45 minutes and told to store the data. In the two motivational-talk treatments, the experimenter added the phrase "Keep up the good work" to all subjects in plenary before leaving the room.

Table 2: Effects of motivation and piece rate on performance

\begin{tabular}{lccc}
\hline \hline & Overall & First half & Second half \\
\hline Motivation & 0.763 & $0.667^{* *}$ & 0.096 \\
& $(0.688)$ & $(0.227)$ & $(0.643)$ \\
Piece rate & $-1.508^{* *}$ & -0.295 & $-1.213^{* *}$ \\
& $(0.568)$ & $(0.242)$ & $(0.347)$ \\
Motivation x piece rate & $3.462^{* *}$ & $0.762^{*}$ & $2.700^{* *}$ \\
& $(0.992)$ & $(0.373)$ & $(0.839)$ \\
Female & $-2.310^{*}$ & $-1.447^{* *}$ & -0.863 \\
& $(1.156)$ & $(0.587)$ & $(0.599)$ \\
Age & $-0.373^{*}$ & $-0.197^{*}$ & -0.175 \\
& $(0.186)$ & $(0.085)$ & $(0.106)$ \\
Enrolled in economics & -0.505 & -0.459 & -0.046 \\
& $(1.064)$ & $(0.526)$ & $(0.641)$ \\
Morning session & 0.772 & 0.364 & 0.409 \\
& $(0.632)$ & $(0.220)$ & $(0.500)$ \\
Constant & $30.97^{* * *}$ & $14.00^{* * *}$ & $16.97^{* * *}$ \\
& $(3.685)$ & $(1.628)$ & $(2.219)$ \\
\hline Observations & 139 & 139 & 139 \\
$R^{2}$ & 0.131 & 0.135 & 0.125 \\
\hline
\end{tabular}

Note: This table reports OLS coefficient estimates (robust standard errors clustered on sessions are given in parentheses). The dependent variable is performance, measured by the number of reported games. "Motivation" is a dummy variable indicating that subjects were exposed to motivational talk. "Piece rate" is a dummy variable indicating subjects earned an additional piece rate. "Motivation x piece rate" is the interaction of both aforementioned dummy variables. We added dummy variables for females, subjects studying economics, and sessions executed in the morning, as well as a control for age. ${ }^{* * *} \mathrm{p}<0.01,{ }^{* *} \mathrm{p}<0.05,{ }^{*} \mathrm{p}<0.1$

Figure 2 depicts the performance for the first and second half of the working period. In all treatments, performance is significantly higher in 
Figure 2: Average performance during first and second half of the working period

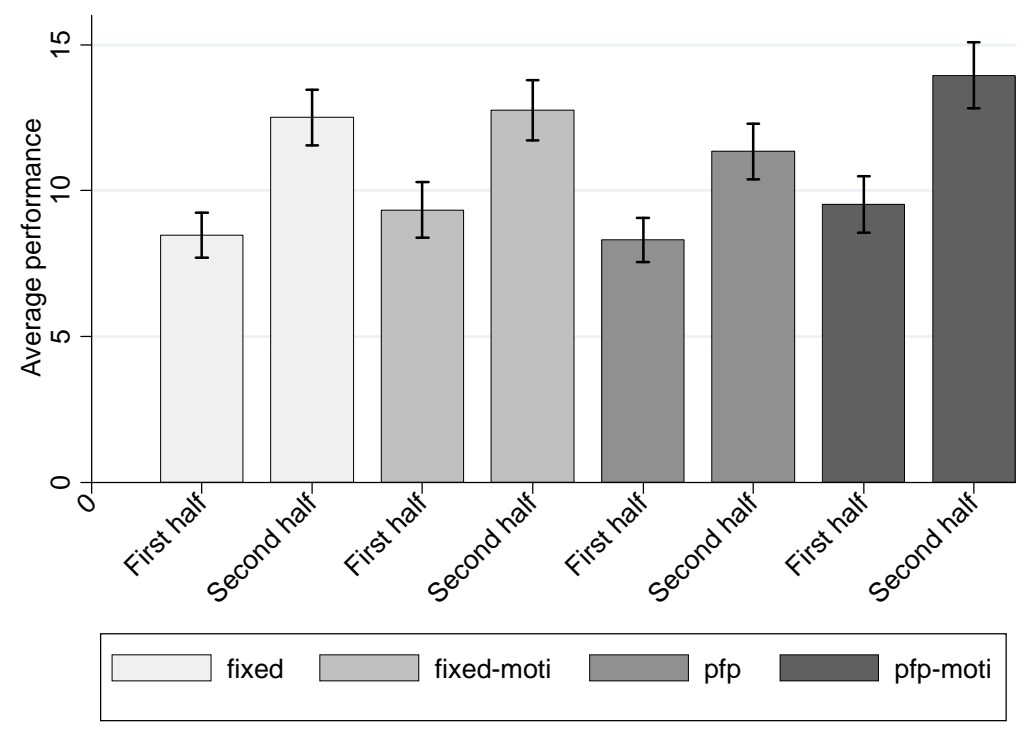

the second half of the working period, which indicates learning effects that are quite common in such tasks (Wilcoxon signed rank test, $p=0.000$ for all treatments).

From the regression in columns (2) and (3) of Table 2, we see that both performance pay and motivational talk alone have weaker motivational effects in the second half than in the first half. Motivational talk alone (without pfp) has a significantly positive effect in the first half, but it disappears in the second half. Performance pay alone does not have a negative effect in the first half, but it becomes significantly negative in the second half. However, the interaction coefficient is positive and significant in both halves, but considerably stronger in the second half. Hence, there is a "hidden benefit of reward" during the whole working period, but it becomes stronger in the second half.

Finally, we investigate how performance pay and motivational talk affect the quality of work. Figure 3 shows the ratio of mistakes (incorrect cell entries) to the number of filled cells for each treatment. ${ }^{16}$ From the bar

\footnotetext{
${ }^{16} \mathrm{We}$ analyze the ratio of mistakes to the number of filled cells to control for the differences in performance between the treatments, but the results are qualitatively
} 
Figure 3: Average ratio of mistakes to number of filled cells

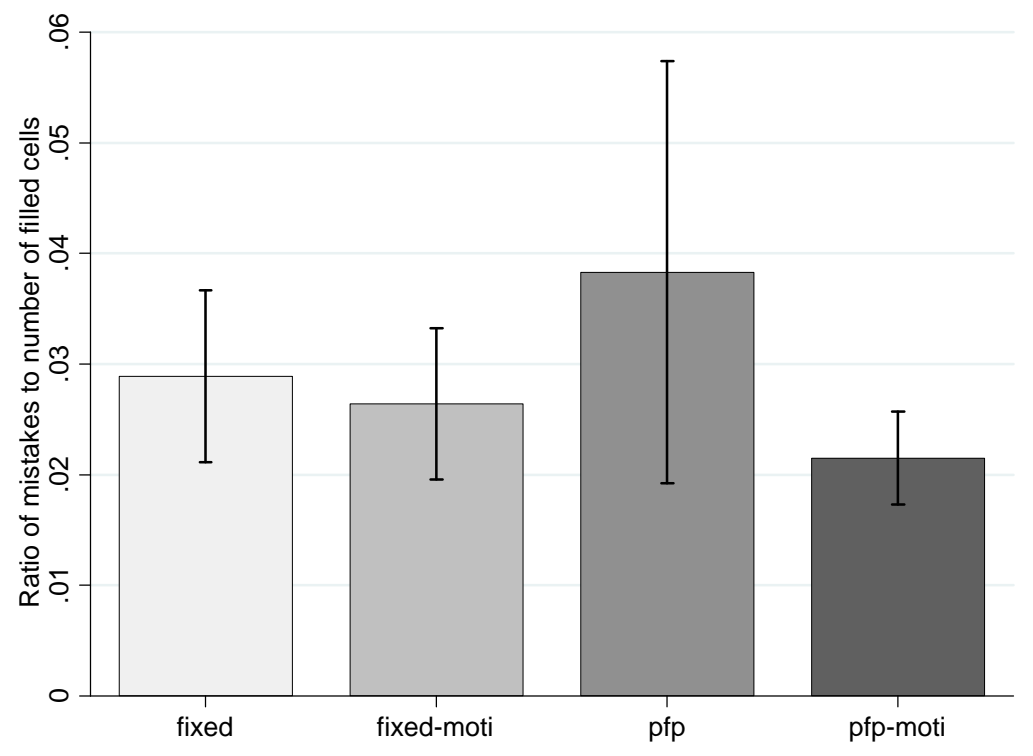

chart in Figure 3 we see that the means are lower when the subjects are exposed to motivational talk. Hence, performance pay alone not only leads to lower performance but also to more mistakes per entered cell. Yet the difference is not significant ( $p=0.169)$, and the regression in column (1) of Table A2 does not show significant effects for the motivation and piece-rate dummies as well as their interaction ( $p$-value for the interaction effect is $0.142)$.

However, when comparing the pfp with the pfp-moti treatment, we observe a significantly lower ratio of mistakes to the number of filled cells in the pfp-moti treatment (F-test for $\beta_{1}+\beta_{3}=0, F=0.015$ ), indicating that indeed adding motivational talk to pay for performance makes the difference. ${ }^{17}$ The average ratio of mistakes to number of filled cells is 0.0383 in the pfp treatment, while the ratio is 0.0215 in the pfp-moti treatment. Hence, adding motivational talk to performance pay leads to a reduction of the mistakes ratio by more than 40 percent. Furthermore, we find significant differences when comparing the fixed-moti with the pfp-

similar if we simply look at mistakes.

${ }^{17}$ The results for regressions with treatment dummies and pfp-moti treatment as the reference category are reported in column (2) of Table A2 in the appendix. 
moti treatment (F-Test for $\beta_{2}+\beta_{3}=0, F=0.036$ ). Hence, although subjects are not rewarded for quality, performance pay has a significantly positive effect on quality if it is accompanied by motivational talk.

Finally, it should be noted that there are huge differences in the variances in the mistakes ratio between treatments. To the extent that variance is of interest here, one could argue that for a given average in the ratio of mistakes, low variance is better than high variance for the overall data quality that the hockey game researchers needed. We find that the variance is highest in the pfp treatment, while it is lowest in the pfp-moti treatment. The variance is in fact thirty times higher in pfp than in pfp-moti (robust test for equality of variances $\mathrm{p}=0.04$ ).

\section{Concluding remarks}

Despite warnings from a number of bestselling business books about the hidden costs of monetary rewards,${ }^{18}$ performance pay is increasingly popular (Lemieux et al., 2009). This might indicate that there exist some hidden benefits of reward, i.e., that monetary incentives interact positively with other motivational tools. In this paper, we present a field experiment designed to investigate the interaction between monetary incentives and a primal form of motivation, namely motivational talk - words that potentially evoke the workers' intrinsic motivation and inspire them to exert extra effort. We identify what can be termed a hidden benefit of reward: Subjects respond to motivational talk by increasing their performance only if they also receive performance pay. Moreover, performance pay decreases performance unless it is accompanied by motivational talk. Consequently, performance pay and motivational talk are complements in enhancing performance. The advantageous combination of performance pay and motivation also carries over to the quality of work. Although subjects were not rewarded for quality, performance pay has a positive effect on quality only if it is accompanied by motivational talk. The effects are strong. In treatments with performance pay, motivational talk increases output by about 20 percent and reduces the ratio of mistakes by more than 40 percent.

\footnotetext{
${ }^{18}$ See, e.g., Nelson (2005) and Pink (2009).
} 
The negative effects of performance pay alone deserve a remark. Although the crowding out effects from monetary incentives are documented in a variety of settings, it is hard to find field evidence on negative effects of piece rates, like we do here. A reason might be that some simple forms of motivational talk are always present in the field, and hence performance pay always interacts with other motivational instruments. In the lab, however, neutral talk or neutral instructions are more common. Our neutral talk does not signal any inherent value of the task nor any prosocial preferences from the principal, and creates an environment in which a small piece rate can have the negative effects that are described in the prominent models of Bénabou and Tirole (2003, 2006) and Ellingsen and Johannesson (2008). But once the principal signals task value or prosociality through motivational talk, we see that the piece rate improves performance rather than backfires. In this respect, our experimental results support the abovementioned theories, and could also inspire a more elaborate version of the multisignal model we propose in the theory section.

Our paper identifies an interaction between monetary incentives and communication. To the extent economists have been interested in communication, it has mainly been related to coordination problems and not to incentives and motivation. But communication is a complex and important variable, with the potential for building bridges between economics and the broader literature on leadership and organizational behavior. More research is needed in order to investigate how different forms of communication interact with different sets of incentive schemes.

Still, the simple experiment presented in this paper has important implications for company practises: First, we show that communication might be crucial to preventing monetary rewards from backfiring. Second, small piece rates, constituting only 10 percent of the total salary, can be rather effective when they are accompanied by simple forms of motivational talk. 


\section{Appendix}

Table A1: Treatment effects on performance

\begin{tabular}{lccc}
\hline \hline & Overall & First half & Second half \\
\hline Fixed treatment & $-2.716^{* * *}$ & $-1.133^{* * *}$ & $-1.583^{* *}$ \\
& $(0.702)$ & $(0.288)$ & $(0.549)$ \\
Fixed-moti treatment & $-1.954^{*}$ & $-0.466^{*}$ & $-1.487^{*}$ \\
& $(0.827)$ & $(0.218)$ & $(0.783)$ \\
Pfp treatment & $-4.225^{* * *}$ & $-1.429^{* * *}$ & $-2.796^{* * *}$ \\
& $(0.539)$ & $(0.274)$ & $(0.442)$ \\
Female & $-2.310^{*}$ & $-1.447^{* *}$ & -0.863 \\
& $(1.156)$ & $(0.587)$ & $(0.599)$ \\
Age & $-0.373^{*}$ & $-0.197^{*}$ & -0.175 \\
& $(0.186)$ & $(0.085)$ & $(0.106)$ \\
Enrolled in economics & -0.505 & -0.459 & -0.046 \\
& $(1.064)$ & $(0.526)$ & $(0.641)$ \\
Morning session & 0.772 & 0.364 & 0.409 \\
& $(0.632)$ & $(0.220)$ & $(0.500)$ \\
Constant & $33.68^{* * *}$ & $15.13^{* * *}$ & $18.55^{* * *}$ \\
& $(4.078)$ & $(1.789)$ & $(2.438)$ \\
\hline Observations & 139 & 139 & 139 \\
$R^{2}$ & 0.131 & 0.135 & 0.125 \\
\hline
\end{tabular}

Note: This table reports OLS coefficient estimates (robust standard errors clustered on sessions are given in parentheses). The dependent variable is performance, measured by the number of reported games. We added dummy variables for each treatment, with the pfp-moti treatment as the reference category. We added dummy variables for females, subjects studying economics, and sessions executed in the morning, as well as a control for age. ${ }^{* * *} \mathrm{p}<0.01,{ }^{* *} \mathrm{p}<0.05,{ }^{*} \mathrm{p}<0.1$ 
Table A2: Effects of motivation and piece rate as well as treatment effects on mistakes

\begin{tabular}{lcc}
\hline \hline & $(1)$ & $(2)$ \\
\hline Motivation & -0.002 & \\
& $(0.007)$ & \\
Piece rate & 0.010 & \\
& $(0.009)$ & \\
Motivation x piece rate & -0.014 & \\
& $(0.008)$ & \\
Fixed treatment & & 0.007 \\
& & $(0.008)$ \\
Fixed-moti treatment & & $0.005^{* *}$ \\
& & $(0.002)$ \\
Pfp treatment & & $0.016^{* *}$ \\
& & $(0.005)$ \\
Female & 0.003 & 0.003 \\
& $(0.002)$ & $(0.002)$ \\
Age & -0.001 & -0.001 \\
Enrolled in economics & $(0.001)$ & $(0.001)$ \\
& -0.004 & -0.004 \\
Morning session & $(0.005)$ & $(0.005)$ \\
& 0.009 & 0.009 \\
Constant & $(0.006)$ & $(0.006)$ \\
& $0.052^{*}$ & $0.052^{*}$ \\
Observations & $(0.022)$ & $(0.022)$ \\
$R^{2}$ & 139 & 139 \\
\hline & 0.064 & 0.064 \\
\hline
\end{tabular}

Note: This table reports OLS coefficient estimates (robust standard errors clustered on sessions are given in parentheses). The dependent variable is the ratio of mistakes to number of filled cells. "Motivation" is a dummy variable indicating that subjects were exposed to motivational talk. "Piece rate" is a dummy variable indicating that subjects earned an additional piece rate. "Motivation x piece rate" is the interaction of both aforementioned dummy variables. In the second column, we added dummy variables for each treatment with the pfp-moti treatment as the reference category. We added dummy variables for females, subjects studying economics, and sessions executed in the morning, as well as a control for age. ${ }^{* * *} \mathrm{p}<0.01,{ }^{* *} \mathrm{p}<0.05,{ }^{*} \mathrm{p}<0.1$ 


\section{Instructions}

The motivational sentences, which were only used in the motivational treatments, are in bold letters.

Dear students,

Welcome to the CIP Pool of the Juridicum. I am glad that you decided to assist us by recording the data for a research project. In the framework of a large research project, we analyze sports data, in this case ice hockey, to investigate the behavior of teams and competitive situations. We are certain that you, as competent students of the University of Bonn, are able to record the data quickly and precisely. My name is XX, and I will explain your task. If you have a question, please raise your hand.

During the next 90 minutes, you are supposed to enter data in an Excel sheet. The Excel sheet is located on a USB flash drive plugged into your computer, and we have already opened it. Please save your work regularly to avoid the loss of data. Please make sure that you do not log out, because all data not stored on the flash drive will be erased. Save your data on the flash drive after the 90 minutes.

To your left, you find a box with game reports from the German ice hockey league. A report can consist of several sheets of paper that are stapled together. Each report has a unique identification number. We will now use an example to show you how the data should be recorded. You find the example game report on the top of the box on your left. Start by entering the ID of the game. You find the ID in the upper-left corner on the first page of the game record. Here, it is $1112 \_358$. The date has to be entered in the second column; here, it is 11.03.2012. You find the date here (showing the date on the screenshot in the powerpoint presentation). Enter the name of the home team into the next column. You find it on the first page denoted as the first team, in this case "Eisbaeren Berlin." Then you enter the visiting team, in this case "Koelner Haie." The number of spectators has to be entered into the next column, and you find this number at the end of the game report in the section "Additional information." Please note that the comma indicates a thousand; hence, we 
have 14,200 spectators. Next you enter the duration of the game, which you can also find in the section "Additional information." Here, you have to enter $02: 25$. Please be careful to enter the duration in the correct format.

Next we have the entries for the number of shot goals for each third. You will find the necessary information in the section "Results" on the first page. Please enter the number of shot goals for the home and visiting teams separately. If the game is tied at the end of the regular time, there will be a so-called overtime. This would be indicated by "OT" in the game report. If there is information available for an overtime, please enter it in the respective columns. In this example, this would be zero for both teams. If a game is still tied after the overtime, there will be a penalty shooting. This would be indicated by "SO" in the game report and would have to be entered in the columns $\mathrm{O}$ and $\mathrm{P}$, as in this example. On the right side of the number of shot goals, you find information regarding shots at goal, which has to be entered next. Again, this information has to be entered for each third and for each team separately. Again, there might be information regarding an overtime or penalty shooting. Next you have to enter the goalie statistics, which you can find on the second page of the game report. Please start with the name of the first goalie of the home team, in this case R. Zepp. His name can be found on the left in the first column. Then you have to enter in the column denoted "MIN" the number of minutes the goalie has been in play. Enter the number of shots at goal and saves into the next columns. If the goalie was changed in a game, the information regarding the second goalie has to be entered in the columns denoted with "Torhueter 2." This is not the case in this example. The same is true for the goalie of the visiting team.

Now we look at the penalties. Here you will have to do some math. There are different kinds of penalties in hockey, and their respective durations depend on the severity of the violation. You find the necessary information on the first page in the section "Penalties." Please start by counting the number of 2-minute penalties of the home team in the first third and then enter the number in column AQ. Here, we have one 2minute penalty. Please enter the number of the penalties and not the minutes; hence, if there was one 2 -minute penalty, you have to enter a "1" 
and not a "2." Next count the number of 5-minute penalties of the home team in the first third and enter it in column AR. We also have 10-minute penalties and 20-minute penalties. For each of those penalties, we have two columns; $10 A$ and $10 D$ and $20 A$ and $20 D$. The automatic 10-minute penalties, which are always called together with a 2-minute penalty, have to be entered in the column $10 \mathrm{~A}$. You can recognize automatic 10-minute penalties because the same player receives an identical penalty of 2 minutes at the exact same time. This is the case in the example game report. $10 D$ penalties are 10-minute penalties that are ruled without an accompanying 2-minute penalty at the same time. Here, you see an example from another game report (shown in the Powerpoint slides). The same logic applies for the 20-minute penalties. Here, you enter the penalty in the column $20 \mathrm{~A}$ if it was ruled together with a 5 -minute penalty. $20 D$ contains all penalties ruled without an accompanying 5-minute penalty. In the column "MP," you enter match penalties with a duration of 25 minutes. Please count the penalties for each team and each third separately and enter the number in the respective column. On your desk you will find a sheet with additional explanations regarding the penalties.

Now we come to the power plays which you can find on the first page of the game report. Enter the first number in the column "goals," here zero, and the second in the column "power," here 5. Next we have the information about the referees and linesmen, which you find at the end of the game report.

When you have finished a game report, please put the corresponding sheets of paper into the box to your right and save the data. Please do not change the order of the game reports. After 45 minutes, I will return and ask you to store the data in an extra file. At that time I will explain to you how exactly this works.

During the next 45 minutes, you will work independently. If you have questions, you can come to the next room and ask me. Of course, you are allowed to leave the room to visit the women's or men's room. After 90 minutes, you will receive your payment of 20 euro in the room next door after you hand in the stick. (In pfp treatments, the following was added: Additionally, we will pay you 10 euro cents for each game you entered. To determine the correct payment we will briefly check the number of games 
you have entered before we pay you.)

I know that the work might be exhausting and tiring, but I also know that you are diligent students who are able to concentrate on these kinds of tasks for longer periods. If you decide to leave the room, please try to be quiet to avoid disturbing your colleagues. You are welcome to adjust the screen to your convenience or to alter the zoom in the excel sheet. If there are no more questions, let's start working! 


\section{References}

Babakus, E., D. W. Cravens, M. Johnston, and W. C. Moncrief (1996). Examining the role of organizational variables in the salesperson job satisfaction model. Journal of Personal Selling and Sales Management 16, $33-46$.

Baldauf, A., D. W. Cravens, and K. Grant (2002). Consequences of sales management control in field sales organizations: A cross-national perspective. International Business Review 11, 577-609.

Bohnet, I., B. S. Frey, and S. Huck (2001). More order with less law: On contract enforcement, trust, and crowding. American Political Science Review 95, 131-144.

Bowles, S. and S. Polanía-Reyes (2012). Economic incentives and social preferences: Substitutes or complements? Journal of Economic Literature 50, 368-425.

Bradler, C., R. Dur, S. Neckermann, and A. Non (2013). Employee recognition and performance: A field experiment. Working Paper.

Brandts, J. and D. J. Cooper (2007). It's what you say, not what you pay: An experimental study of manager-employee relationships in overcoming coordination failure. Journal of the European Economic Association 5, $1223-1268$.

Bénabou, R. and J. Tirole (2003). Intrinsic and extrinsic motivation. Review of Economic Studies 70, 489-520.

Bénabou, R. and J. Tirole (2006). Incentives and prosocial behavior. American Economic Review 96, 1652-1678.

Cameron and Pierce (1994). Reinforcement, reward, and intrinsic motivation: A meta-analysis. Review of Educational Research 64, 363-423.

Deci, E. (1975). Intrinsic Motivation. New York: Plenum Press.

Deci, E. L. (1971). Effects of externally mediated rewards on intrinsic motivation. Journal of Personality and Social Psychology 18, 105-115. 
Deci, E. L. and R. M. Ryan (1971). Effects of externally mediated rewards on intrinsic motivation. Journal of Personality and Social Psychology 18, $105-115$.

DelVecchio, S. and J. Wagner (2011). Motivation and monetary incentives: A closer look. Journal of Management and Marketing Research 7, 1-13.

Dur, R., A. Non, and H. Roelfsema (2010). Reciprocity and incentive pay in the workplace. Journal of Economic Psychology 31, 676-686.

Ellingsen, T. and M. Johannesson (2008). Pride and prejudice: The human side of incentive theory. American Economic Review 98, 990-1008.

Fehr, E. and B. Rockenbach (2003). Detrimental effects of sanctions on human altruism. Nature 422, 137-140.

Frey, B. S. and F. Oberholzer-Gee (1997). The cost of price incentives: An empirical analysis of motivation crowding-out. American Economic Review 87, 746-755.

Gneezy, U., S. M. and P. Rey-Biel (2011). When and why incentives (don't) work to modify behavior. Journal of Economic Perspectives 25, 191-210.

Gneezy, U. and A. Rustichini (2000a). Pay enough or don't pay at all. Quarterly Journal of Economics 115, 791-810.

Gneezy, U. and A. Rustichini (2000b). A fine is a price. Journal of Legal Studies 29, 1-18.

Grant, A. M. and F. Gino (2010). A little thanks goes a long way: Explaining why gratitude expressions motivate prosocial behavior. Journal of Personality and Social Psychology 98, 946-955.

Henning-Schmidt, H., B. Rockenbach, and A. Sadrieh (2010). In the search of workers real effort reciprocity - a field and a laboratory experiment. Journal of the European Economic Association 8, 817-837.

Kosfeld, M. and S. Neckermann (2011). Getting more work for nothing? symbolic awards and worker performance. American Economic Journal: Microeconomics 3, 86-99. 
Kube, S., C. Puppe, and M. A. Maréchal (2012). The currency of reciprocity - gift-exchange in the workplace. American Economic Review 102, 1644-1692.

Kvaløy, O. and A. Schöttner (2013). Incentives to motivate. Working Paper.

Lemieux, T., W. B. MacLeod, and D. Parent (2009). Performance pay and wage inequality. Quarterly Journal of Economics 124, 1-49.

Lepper, M. R. and D. Greene (1978). The Hidden Costs of Reward: New Perspectives on the Psychology of Human Motivation. Lawrence Erlbaum.

Marino, A. M. and J. Zábojnik (2008). Work-related perks, agency problems, and optimal incentive contracts. Rand Journal of Economics 39, $565-585$.

Miao, C. F. and K. R. Evans (2007). The impact of salesperson motivation on role perceptions and job performance - a cognitive and affective perspective. Journal of Personal Selling and Sales Management 27, 89-101.

Nelson (2005). 1001 Ways to Reward Employees. Workman Pub.

Pink, D. H. (2009). Drive - The Surprising Truth About What Motivates Us. Riverhead Trade.

Pokorny, K. (2008). Pay - but do not pay too much. an experimental study on the impact of incentives. Journal of Economic Behavior \&3 Organization 66, $251-264$.

Robbins, S. P. and T. A. Judge (2013). Organizational Behavior. Pearson Education Limited, Harlow.

Sims, R. R. (1998). Reinventing training and development. Quorum books.

Sliwka (2007). Trust as a signal of a social norm and the hidden costs of incentive schemes. American Economic Review 97, 999-1012. 
Stajkovic, A. D. and F. Luthans (2003). Behavioral management and task performance in organizations: Conceptual background, meta-analysis, and test of alternative models. Personnel Psychology 56, 155-194. 